\title{
Is Liquid Water a Hot Quantum Fluid? Anomalies of Water in Thin Liquid Films and in Biological Systems
}

\author{
Byoung Jip Yoon \\ Department of Chemistry, Kangnung National Universin, Kangnung, Kangwondo 210-702. Korea \\ Received Mav 10, 2003
}

\begin{abstract}
The anomalies that appear at every multiple of $15^{\circ} \mathrm{C}$ in the viscosity of a thin liquid film of water and of water near solid interfaces are explained in this paper by conparing the thermal wavelength and molecular free volume of water, and quantum numbers are found. The possibility that these anomalies are related to the preferred and/or lethal temperatures of organisms is considered. The toxicity of heavy water ( $\left.D_{-} \mathrm{O}\right)$ can also be explained with this approach
\end{abstract}

Key Words : Water. Hot quantum fluid. Thin liquid film. Anomalies of water. Toxicity of heavy water

\section{Introduction}

Knowledge of the properties and structure of water is crucial to understanding various biological phenomena. Water is a typical liquid. but in contrast to other simple liquids has many mysterious properties. For instance the density of liquid phase of water is higher than the density of its crystalline phase. ice. The density maximum of water appears near 4 " $\mathrm{C}$ and accordingly below $4{ }^{\circ} \mathrm{C}$ its thermal expansion coefficient is negative. The magnitude and temperature dependence of compressibility of water is not easily reproduced. on a single molecule basis. ${ }^{1}$ The heat capacity of water is about twice that of ice. which is surprising because thermal energy is usually stored in the vibrational modes and the intermolecular vibrational frequencies of water are similar to those of ice. ${ }^{2}$ Further. considering water's high hydrogen bond energy: water is not very viscous nor does it have a high surface tension. Also mysterious is observation that about $10 \%$ of hydrogen bonds are broken ${ }^{3}$ at the melting point despite the high energy of the hydrogen bond intermolecular interaction. which is about ten times larger than the thermal kinetic energy. Explanations of these anomalies have been attempted by many authors but no model explicitly explaining all the anomalies has appeared so far. ${ }^{+}$

Water in thin liquid films also exhibits anomalous behavior. Reports indicate that the viscosity of water between two quartz plates separated by several hundred angstroms shows a periodic nature with peaks at every multiple of $15^{\circ} \mathrm{C} .{ }^{5}$ The maximum viscosity of this variation is about twice the minimum viscosity. The variation with temperature of the disjoining pressure of a $100-\AA$ layer of water between two quartz plates has been measured: inflections at about the same temperatures (i.e. multiples of $15{ }^{\circ} \mathrm{C}$ ) have been observed. ${ }^{67}$ The viscosity of water confined more narrowly to subnanometre films was measured at $23^{\circ} \mathrm{C}^{8}$ The temperature dependence of the diffusion coefficient of thiourea in

\footnotetext{
*Corresponding author. Tel: +82-33-640-2301, Fax: +82-33-6471183 , e-mail: bivoon $t$ kangnung ac. $\mathrm{kr}$
}

water has kinks at approximately $15,30,45.60^{\circ} \mathrm{C}$. that is at multiples of $15^{\circ} \mathrm{C}$. Various other examples for anomalies at these temperatures are near solid interfaces. ${ }^{10}$ The anomalies of thin film of liquid water or of water near interfaces seem to be related to the properties of water in cells and therefore to the preferred temperatures of animals or plants (i.e. to the body temperature for homoiothermic animals). or relatedly to the temperatures that are lethal for these organisms. "The body temperature of mammals is $37^{\circ} \mathrm{C}$. i.e. halfway between 30 and $45^{\circ} \mathrm{C}$. The preferred temperature for heterothermic organisms such as insects. fish. soil bacteria and mould is about $23^{\circ} \mathrm{C} \cdot{ }^{11}$ i.e.. halfway between 15 and $30^{\circ} \mathrm{C}$. Yeasts are usually cultured at $24^{\circ} \mathrm{C}^{12}$ Of course there are variations in lethal temperatures. The upper lethal temperature of alga is between 31.5 and $32.5^{\circ} \mathrm{C}$ and the lower lethal temperature between 9 and $10^{\circ} \mathrm{C} .^{13}$ The body temperatures of dog. cat. and pigeon are 39. 38.5 and $41{ }^{\circ} \mathrm{C}$. which are not quite halfway but avoid 30 and $45^{\circ} \mathrm{C}$. At around $15^{\circ} \mathrm{C}$. plants are low-temperature damaged. ${ }^{11}$ Pasteurization is often carried out at $60^{\circ} \mathrm{C}$. This tendency of organisms to avoid temperatures that are multiples of $15^{\circ} \mathrm{C}$ might be related to the structure of water in small cells and thus to the anomalies of thin liquid film of water. The viscosity of water in thin liquid film or water confined to a small space sharply increases at temperatures that are multiples of $15^{\circ} \mathrm{C}$ and thus at these temperatures metabolites will not be easily transported within cells. which will result in many other biochemical effects.

\section{Theory and Calculations}

Molecules in liquid phase typically display thermal motion that takes up a larger volume than such motion in the solid phase. Monatomic simple liquids like argon expand about $12 \%$ in volume at the melting point or that amount of free volume is included. This free volume is related directly to the entropy: At high temperature. when a molecule is in thermal motion, the thermal wavelength. $\lambda_{T}$. is defined and it is referred to as the effective size of the molecule. as follows. 


$$
\lambda_{T}=\frac{h}{\sqrt{2 \pi m k T}}
$$

in which the terms have their usual statistical mechanical significances. The thermal wavelength of an ordinary molecule at room temperature is very small compared with the size of molecular free volume. Therefore at room temperature molecules can be thought of as moving freely in free space. However when the temperature is low and the thermal wavelength is long. or when the molecular free volume is relatively small even at high temperature. quantum effects take place due to the long thermal wavelength.

The intermolecular interaction between water molecules is very strong. Therefore large holes or vacancies are not expected in the liquid phase of water. According to calculations using the significant structure theory, the free volume of liquid water is very small. ${ }^{1+}$ There have been some controversies about the philosophy of this approach. which is based on a free-rolume theory. but it reproduces various thermodynamic properties of many liquids using reasonable and physically meaningful parameters. ${ }^{15}$ When this theory is applied to liquid water. it provides satisfactory estimates of water's molar volume. vapor pressure. entropy and entropy of vaporization (see Table 1). This success indicates that the dependence of the free energy of water on temperature and volume. or on the free volume. is properly modeled by this theory. The values of the estimated free volume at various temperatures are listed in Table 1 . When the one-dimensional molecular free volume $\left(l_{f}=\left(v_{f}\right)^{13}\right.$. where $v_{f}$ is the free volume in molecular dimension) is divided by the thermal wavelength. the quantum numbers $\left(q=l_{f} / \dot{\lambda}_{T}\right)$ appear at approximate half integers for the following temperatures: 15 ${ }^{\circ} \mathrm{C}(q=2.9) .30^{\circ} \mathrm{C}(3.7) .45^{\circ} \mathrm{C}(4.2)$, and $60^{\circ} \mathrm{C}$ (4.8). The calculated entropies are somewhat higher than the experimental values. so it seems that the estimated free volumes are also slightly too high. Thus the correct $q$ values are probably closer than calculated to half integers at temperatures that are multiples of $15^{\circ} \mathrm{C}$

In instances for which the significant structure model is unsatisfactory and efforts to set up the partition function and

Table 1. Free volumes and quantum numbers of $\mathrm{H}_{2} \mathrm{O}$

\begin{tabular}{|c|c|c|c|c|c|c|c|}
\hline $\begin{array}{c}t \\
(" \mathrm{C})\end{array}$ & $\begin{array}{l}\lambda_{T} \\
\AA\end{array}$ & $\left(\begin{array}{c}y \\
\left(\mathrm{~cm}^{3} / 1 \mathrm{nol}\right)\end{array}\right.$ & $\begin{array}{c}P \\
\text { (atm) }\end{array}$ & $\begin{array}{l}v / v \\
(\%)\end{array}$ & $q$ & $\mathrm{Ifiet}_{\mathrm{O}} \mathrm{OH}$ & $\begin{array}{c}S \\
\text { (eu) }\end{array}$ \\
\hline 15 & 0.242 & $\begin{array}{c}18.036 \\
(18.032)\end{array}$ & $\begin{array}{c}0.017 \\
(0.017)\end{array}$ & 1.1 & 2.9 & 0.11 & $\begin{array}{c}16.4 \\
(16.1)\end{array}$ \\
\hline 30 & 0.236 & $\begin{array}{c}18.096 \\
(18.095)\end{array}$ & $\begin{array}{c}0.043 \\
(0.042)\end{array}$ & 2.2 & 3.7 & 0.13 & $\begin{array}{c}17.5 \\
(17.0)\end{array}$ \\
\hline 45 & 0.231 & $\begin{array}{c}18.195 \\
(18.194)\end{array}$ & $\begin{array}{c}0.097 \\
(0.095)\end{array}$ & 3.1 & 4.2 & 0.14 & $\begin{array}{c}18.3 \\
(17.9)\end{array}$ \\
\hline 60 & 0.225 & $\begin{array}{c}18.331 \\
(18.324)\end{array}$ & $\begin{array}{c}0.203 \\
(0.200)\end{array}$ & 4.1 & 4.8 & 0.16 & $\begin{array}{c}19.1 \\
(18.7)\end{array}$ \\
\hline
\end{tabular}

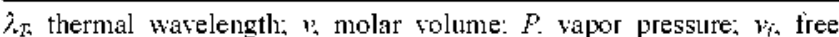

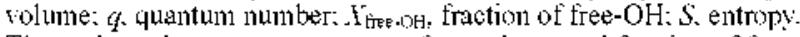

The nolar volume, vapor pressure. free volume and fraction of free- $\mathrm{OH}$ are calculated using the significant structure model of water. 14. Observed values are in parentheses. parameterization become too complicated. ${ }^{1+4}$ a simpler approach is possible. According to the free volume theory of Kirkood. ${ }^{16.17}$ the entropy. $S$ is expressed as follows.

$$
\frac{S}{R}=C_{i j}+\ln \frac{y_{j}}{\lambda_{T}^{3}}
$$

where $R$ is the gas constant. This equation is equivalent to the expression for the entropy of an ideal gas if $C_{0}$ is $5 / 2$ and $v_{i f}$ is the molecular volume. Equation (2) seems too simple to apply to liquid water, but if non-ideal effects are included in the dependence of the free volume on temperature. this equation provides a reasonable method for estimating the temperature dependence of water's entropy. The contribution to entropy from intramolecular vibrations is so small as to be negligible. Using the experimental value for the entropy and $q=3.0$ at $15^{\circ} \mathrm{C}$ (estimated from Table 1), $C_{c}=4.82$ is obtained. This is very similar to the value obtained. when the entropy of ice at $0^{\circ} \mathrm{C}$ is divided by the gas constant, $R$, i.e., $C_{0}=4.85$. Given this value for $C_{\text {. }}$. the free volumes and quantum numbers are calculated and are listed in Table 2. Using this approach, the quantum numbers also take on half integer or integer at every multiple of $15^{\circ} \mathrm{C}$. At the temperatures where the quantum number is a multiple of half integer, the nodes of themal wavelength lie on the boundary of molecular free space, or in other words, the effective size of a molecule in thermal motion coincides with the molecular free volume (see Figure 1). Therefore molecular motions will be stationary or commensulrate at these temperatures and thus a high viscosity is expected. On the other hand when the nodes do not lie at the boundary of molecular free space, the molecule moves more freely and can enter neighboring space resulting in a low viscosity. This explains the fact that water has unusually large root-meansquare fluctuations in density and in energ. ${ }^{1,2}$ which accounts for its large compressibility and heat capacity. Such entry into neighboring space may occur in the bulk phase of water without any increase in total molar volume because of manybody cooperative motion or because of the softness of the boundaries of molecular free space. However. in confined spaces or at a boundary of heavy rigid molecules. these effects manifest as macroscopic themodynamic properties. This limitation of molecular movement amounts to a repulsion between molecules that may also result in the breaking of more lydrogen bonds. In Eq. (2), only the

Table 2. The quantum numbers and free volumes calculated with Eq. (2)

\begin{tabular}{cccc}
\hline$t(\mathrm{C})$ & $q$ & $v_{f}\left(\mathrm{~cm}^{3 / 2} / \mathrm{mol}\right)$ & $\alpha_{s}$ \\
\hline 0 & 2.48 & 0.142 & -2.6 \\
15 & 3.00 & 0.232 & -2.3 \\
30 & 3.50 & 0.341 & -2.0 \\
45 & 4.05 & 0.491 & -2.0 \\
60 & 4.66 & 0.696 & -2.2 \\
\hline
\end{tabular}

$q$ quantum number; $v_{4}$ free volume; $\left(x_{34}\right.$ themal expansion coefticient of intrinsic water molecule estimated from the experimental entropy value. 
(a)

(b)

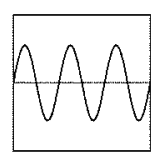

(c)

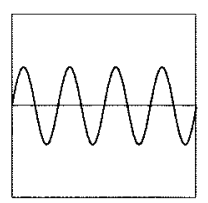

(d)

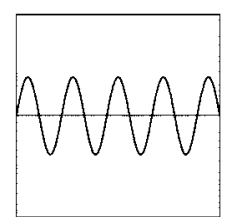

Figure 1. The relationship between the thennal wavelength and the molecular free space. The nodes of themal wavelength lie on the boundary of molecular free space, resulting in stationary wave functions. (a) $15^{\circ} \mathrm{C}(q=3.0)$, (b) $30^{\circ} \mathrm{C}(q=3.5)$, (c) $45^{\circ} \mathrm{C}$ $(q=4.0)$, and $(d) 60^{\circ} \mathrm{C}(q=4.5)$. These quantum number arise for heavy water are at $19,32,44,56{ }^{\circ} \mathrm{C}$, respectively. The same phenomena are at the melting point $(q=2.5)$, and at $-16(q=2.0)$ and $-36^{\circ} \mathrm{C}(q=1.5)$ in the supercooled region.

translational degree of freedom (or vibration in lattice free space) is described. but the molecule has its own intrinsic volume $v_{s}$. Therefore the total molecular volume. $v$ of the liquid can be expressed as.

$$
v=v_{s}+v_{f}
$$

and

$$
\alpha=\frac{v_{s}}{v} \alpha_{s}+\frac{v_{f}}{v} \alpha_{f}
$$

in which $\alpha$ is the thermal expansion coefficient and the subscripts $s$ and $f$ denote intrinsic volume and free volume. respectively. $\alpha_{s}$ is calculated using the experimental value for $\alpha$ and estimated free volume: and these values are listed in the last column of Table 2 . These values are negative indicating that the effective intrinsic volume of water slurinks with rising temperature. This can be explained in two ways: Firstly perhaps a change from an open structure to a dense structure is involved. This idea has often been used as a twostructure mixture model. ${ }^{1.14}$ Secondly perhaps the effective intermolecular potential (including many body interactions with quantum mechanical correction) is soft and so the repulsive hard core of the intermolecular interaction potential shrinks with increases in the kinetic energy. This effect is enhanced by the decrease in the quantum repulsion that results from any mismatch between the thermal wavelength and molecular free space. which is not usually included in two-body intermolecular potentials typically used in simulations. It has previously been shown that quantum mechanical corrections to the molecular interactions of water make them softer than is predicted classically. ${ }^{18.15}$ The value $\alpha_{5}=-2.0 \times 10^{-4} / \mathrm{K}$ corresponds to a slope of repulsive soft core of $r \partial u(r) / \partial r=187 \mathrm{~kJ} / \mathrm{mol}$ (when the interaction energy increases due to the thermal kinetic energy of $3 / 2$ kT). This value for $\alpha_{s}$ also corresponds to the repulsion at $r=$ $2.78 \mathrm{~A}$ for the spherically averaged potential of water $(\varepsilon / k=$ $2400 \mathrm{~K}$ and $\sigma=2.65 \AA\left[r_{m}=2.97 \AA\right]$ in a Lennard-Jones form) ${ }^{19}$ while $R_{\circ 0}$ in the liquid phase of water is about 2.8 A. The water molecule is itself not sufficiently soft or flexible to account for this negative value of $\alpha_{s}=21$

The density maximum of water at $4{ }^{\circ} \mathrm{C}$ has often been discussed $=3$ it is typically explained by the conjunction of

the decrease in intrinsic volume (termed in this paper) with the increase in free volume. The fact that water freezes at 0 ${ }^{\circ} \mathrm{C}$ can then be made sense of. because it may be that at this temperature water has a quantum number $(q=2.5)$. Extending this idea to supercooled water. singular temperatures at which water might easily freeze should occur at around -16 ${ }^{\circ} \mathrm{C}(q=2.0)$ and $-36^{\circ} \mathrm{C}(q=1.5)$ which correspond to the historic limits of supercooling ${ }^{24.25}$ If this quantum mechanical approach is plausible. then it can be predicted that the preferred temperature of organisms will not occur near multiples of $15^{\circ} \mathrm{C}$. since at these temperatures the thermal motions of water molecules are stationary. which makes the transport of metabolites in cells difficult. The cell wall and other heavy materials in cells are likely to act as boundaries for the thermal wavelength of the water molecules. even though cells are not typically small enough to exhibit quantum effects. Many vegetables and fruit are preserved for long-term storage at $0^{\circ} \mathrm{C} .^{26}$ According to this idea. the temperature $15^{\circ} \mathrm{C}$ will also keep them fresh. because of the low activity of water molecules at these temperatures. The preferred temperature for preserving fruit or vegetables such as apple. grape. or strawberry is $0-2^{\circ} \mathrm{C}$ and that for banana. tomato. and sweet potato is $13-16^{\circ} \mathrm{C}^{26}$

\section{Application to Heavy Water}

The thermodynamic properties of heavy water are different to those of $\mathrm{H}_{2} \mathrm{O}$. because of the variation of quantum effects with mass. Equation (2) can be applied to heavy water using the experimental value of entropy for $\mathrm{D}_{2} \mathrm{O}$ and assuming a value for $\mathrm{C}_{i j}$ of 5.60, i.e.. the value of the entropy of $\mathrm{D}_{2} \mathrm{O}$ ice at its melting point divided by $R$. And the quantum numbers are found. As a series of $\left.\left(r{ }^{\circ} \mathrm{C}\right), q\right)$, the results are $(4,2.5)$. $(19.3 .0)$. (32.3.5). (44.4.0). and (56.4.5). Thus in the case of heavy water. a quantum number of $q=2.5$ is found at the melting point. The thermal wavelength of heavy water is $95 \%$ shorter than that of water at the same temperature. Thus the temperature interval is smaller for heavy water than for $\mathrm{H}_{2} \mathrm{O}$. The toxicity of heavy water has been widely reported ${ }^{27}$ This toxicity is not a difference in chemistry but perhaps can be explained as follows. The thermal wavelength of $\mathrm{D}_{2} \mathrm{O}$ or $\mathrm{HOD}$ is shorter than that of $\mathrm{H}_{2} \mathrm{O}$. Thus the ingestion of heavy water by organisms has a similar effect to raising the body temperature or to increasing the viscosity within cells at their preferred temperatures. It is curious that $\mathrm{D}_{2} \mathrm{O}$ has the higher viscosity in bulk phase than $\mathrm{H}_{2} \mathrm{O}^{28}$ despite its higher percentage of broken hydrogen bonds. ${ }^{3}$ This effect might be also due to a weaker quantum mechanical repulsion in $\mathrm{D}_{2} \mathrm{O}$ than arises in $\mathrm{H}_{2} \mathrm{O}$ and because the spherically averaged effective potential of $\mathrm{D}_{2} \mathrm{O}$ is broader than that of $\mathrm{H}_{2} \mathrm{O}$ because of its lower intermolecular vibrational frequencies. $\mathrm{D}_{2} \mathrm{O}$ is also useful for hypothermic storage. ${ }^{29}$ probably because of its high viscosity.

\section{Conclusion}

A strong hydrogen bond interaction occurs between water 
molecules. Thus water molecules do not easily separate and therefore the small free volume is included. In this small free space the water molecule exhibits high-temperature quantum mechanical behavior. The water is the lightest molecule of all liquids that exist at room temperature and atmospheric pressure. The anomalies in a thin liquid film of water are thus not due to a sufface effect derived from the surface/ volume ratio. but rather to a wall effect produced by boundaries. resulting in quantum mechanical repulsion between molecules.

Acknowledgement. This work was supported in part by Kangnung National Universiț:

\section{References}

1. Frank; H. S. In Water A Comprehensive Treatise: Franks. F, Ed: Plenum: New York. 1975: Vol. 1. Chap. 14

2. Eisenberg. D.: Kauzmann. W. The Structure and Properties of Water: Onford Univ. Press: New York. 1969: pp 173-181.

3. Luck, W. A. P; Ditter, W. J. Phys Chent 1970. 74, 3687-3695.

4. Cho, C. H.: Singh. S. Robinson. G. W. J. Chem. Plns. 1997. 107, $1-10$.

5. Peschel. G.: Aldtinger. K. H. Natumwiss. 1969. 56. 558.

6. Clifford. J. in Ref. 1. Vol. 5. Chap. 2. p 108.

7. Peschel. G.: Aldtinger. K. H. Natwwiss. 1967. 54.614.

8. Raviv, U. Laurat P. Klein, J. Nature 2001, H3. 51-54

9. Drever. G.: Kahrig. E.: Kirstein. D.: Erpenbeck; J: Lange. Fr. Xaturwiss. 1969, 56, 558 .
10. Dorst-Hansen, Indust. Eng. Chem. 1969. 61, 10-47.

11. Uedaira, H. What is Water? (in Japanesej. Blue Backs Series; Koudan Press: Tohyo. 1973: Vol. B-335.p 175.

12. ATCCC Cat. Yeasts. 8th ed.: Amer. Type Culture Collection. 1990.

13. Komatsu. T:- Meinesz, A :- Buckles. D. MEPS 1997, 1+6. 145-153.

14. Yoon, B. J.: Jhon, M. S. Studies in Phys. Theor Chem. 1982, 27. 29-44.

15. Eyring. H.: Thon. M. S. Significant Liquid Stucturs. Wiley: New York. 1969.

16. Kirkood. J. G. J. Chen. Pho 1950. 18. 380-382.

17. Hirschfelder, J. O; Curtiss, C. F.: Bird. R. B. Molecular Theory of Gases and Liquids. Wiley: New York, 1954: pp 272-285.

18. Kuharski, R. A.: Rossky. P. J. Chem. Phys. Lett. 1984, $103,357$. 362

19. Yoon. B. J.: Thon. M. S.: Scheraga. H. A. J. Chem. Phus. 1990.92. $3748-3755$

20. Yoon, B. J.; Morokuma. K.; Davidson. E. R. J. Chent Phys. 1985. 83. $1223-1231$

21. Yoon, B. J.; Jhon. M. S. Chem. Phus. Lett. 1991. 178. $253-258$.

22. Cho. C. H.: Singh. S.: Robinson. G. W. Phus. Rent Lett. 1996. 76. 1651-1654.

23. Velasco. E.: Mederos. L.: Navascus. G. Phns. Ren: Lell. 1997. 79. 179 .

24. Rasmussen. D. H.: MacKenzie, A. P. Angell. C. A.: Tucker, J. C. Science 1973.181, 342-344.

25. Bottomley. G. A. Aust. J. Chem 1978. 31.1177-1180.

26. Ginsburg. L. Deciduous Fruit Grower 1965. 15. 80-86.

27. Kushner. D. J: Balcer. A.: Dunstall. T. G. Cam. J. Phsiol. Phamacol 1999, 77. 79-88.

28. Kell, G. S. Chap 10 in Ref. 1

29. Berwanger, C. S. et al Transplantation 1998. 65.735-737. 